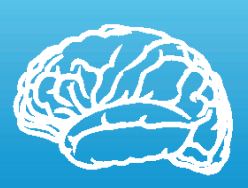

NELROLOG
Department of Oral Medicine and Radiology, Manipal College of Dental Sciences, Manipal, Manipal Academy of Higher Education, Manipal, Karnataka, India
DOI: $10.15386 / \mathrm{mpr}-1389$

Manuscript received: 07.06.2019

Received in revised form: 03.09.2019

Accepted: 30.09.2019

Address for correspondence:

shruthi.acharya@manipal.edu

This work is licensed under a Creative Commons Attribution-NonCommercialNoDerivatives 4.0 International License

\title{
Bilateral Bell's palsy in a young female: a rare case report
}

Mathangi Kumar, Shruthi Acharya, Ravindranath Vineetha, Keerthilatha M. Pai

\begin{abstract}
Bell's palsy is described as a type of facial paralysis, which is unilateral in $70 \%$ of the cases. Bell's palsy accounts for about $23 \%$ of bilateral facial paralysis. Here, we present a rare case of bilateral Bell's palsy in a 15-year-old female who developed sudden facial weakness with no associated symptoms. The patient was subjected to thorough clinical, laboratory, and necessary radiological investigations. As there was no conclusive evidence from any of the investigations, this could be a case of idiopathic bilateral Bell's palsy. This is the first ever case of bilateral Bell's palsy in a young female which has been reported in the Indian population.
\end{abstract}

Keywords: facial palsy, pediatric, idiopathic, Lyme disease, facial pain

\section{Introduction}

Bilateral facial palsy is an uncommon condition, which is caused due to infra-nuclear lesions. Lower motor neuron palsy is termed as Bell's palsy. Bell's palsy is a type of facial paralysis, which is unilateral in $70 \%$ of the cases. Bell's palsy accounts to about $23 \%$ of bilateral facial paralysis [1]. Here we present a rare case of bilateral Bell's palsy in a young female who developed sudden facial weakness with no associated symptoms. The patient was subjected to thorough clinical, laboratory, and necessary radiological investigations.

\section{Case report}

A 15-year-old female reported a sudden onset of pain behind the left ear for the last two days. She complained of redness, foreign body sensation in the eyes for 2 months along with slurring of speech, inability to close both eyes completely, smile and frown. Patient reported that the facial weakness initially started on the right side, which progressed to involve the left side of the face after three weeks. Her medical history was non-contributory for any systemic disorder. She had sought consultation at other centers where she was given TENS therapy (Transcutaneous Electric Nerve Stimulation) which provided no relief of symptoms. There was no history of any recent fever, myalgia, sore throat, headache, skin rashes, preceding trauma, numbness of the face, or similar episodes of facial weakness in the past.

On clinical examination, the patient was afebrile, and she was unable to close her eyes completely. Bell's sign was positive (Figure 1). There was absence of wrinkling of forehead, inability to hold air in the mouth / blow the cheeks. Sensory examination revealed no sensory deficits. Deviation of the corner of mouth to the left side was noted. Bilateral submandibular lymph nodes were soft and mobile. We established a clinical diagnosis of bilateral Bell's palsy and gave an immediate referral to Neurology for expert management. Under hospitalization, following investigations were advised and reported as follows: Laboratory tests (red blood cell count, white blood cell count, hemoglobin) were normal; Angiotensin Converting Enzyme (ACE) levels were normal ruling out sarcoidosis. Cerebrospinal fluid (CSF) analysis revealed clear fluid with 
protein levels $(24.0 \mathrm{mg} / \mathrm{dL})$, glucose levels $(3.6 \mathrm{mmol} / \mathrm{L})$, chloride levels $(120 \mathrm{mmol} / \mathrm{L})$ and culture was negative after 60 hours of incubation, and India Ink staining did not show any morphological forms which could indicate Cryptococcal infection. Enzyme Linked Immunosorbent Assay (ELISA) for JEV (Japanese Encephalitis Virus) was negative. Plain Magnetic Resonance Imaging (MRI) (GE Signa Excite ${ }^{\mathrm{TM}} 1.5 \mathrm{~T}$ Scanner) of brain was normal with no compression of the cisternal segment of bilateral facial nerves on axial sections (Figure 2). Audiological evaluation suggested that the hearing sensitivity was within the normal range in both the ears. Minor salivary gland tissue biopsy did not reveal features of Sjogren's syndrome.

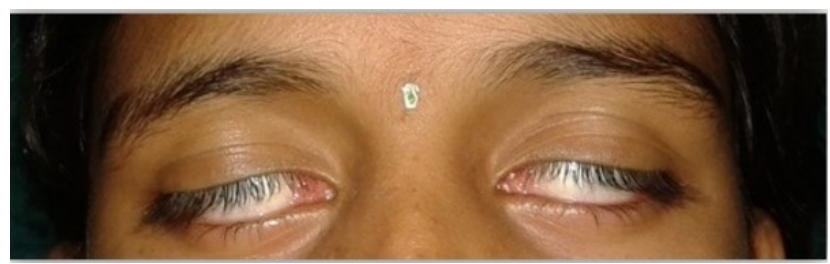

Figure 1. Photograph showing positive Bell's sign.

Ultrasonography (USG) of the neck showed cervical lymphadenopathy, and FNAC (Fine needle aspiration cytology) of the lymph node was suggestive of reactive lymphadenitis. Ophthalmological examination revealed intact corneal reflexes and Schirmer's test was normal. The symptomatic treatment was Tab. Prednisolone $10 \mathrm{mg}$ once a day and Tab. Acyclovir $400 \mathrm{mg}$ thrice daily for a week. Supportive treatment in the form of physiotherapy and facial nerve stimulation was performed. Teardrops every 2 hours and protective eyewear were given and she was discharged. She was then advised to taper the steroid dosage on the next follow up. The facial weakness gradually waned and patient was completely to her normal self after a month with no residual neurological deficits.

\section{Discussion}

The occurrence of bilateral facial palsy is exceedingly rare $(0.3-2 \%$ of all facial palsies) with an incidence of only one in 5,000,000 population [2]. Lyme's disease represents $50 \%$ of cases of bilateral facial palsy in children [3]. This condition often poses a diagnostic challenge, particularly when it presents in young individuals as in the present case. The various causes of facial palsy are listed in Table I.

In acute HIV-1 infection, transient facial nerve paralysis precedes seroconversion by $4-6$ weeks, thus facilitating early diagnosis. The clinician's suspicion should be further raised with the presence of bilateral facial nerve paralysis, a recent flu-like illness, and Ramsay-Hunt syndrome with disseminated herpes zoster [3].

Lyme disease; caused by Borrelia burgdorferi is a spirochete infection with common ticks as the carrier $[9,10]$. The disease typically goes through three phases: initial stage of fever, headache, followed by meningitis, encephalitis, and final stage of debilitating arthritis and chronic CNS disease. Therefore, it is important to recognize the cause for facial palsy in pediatric population.

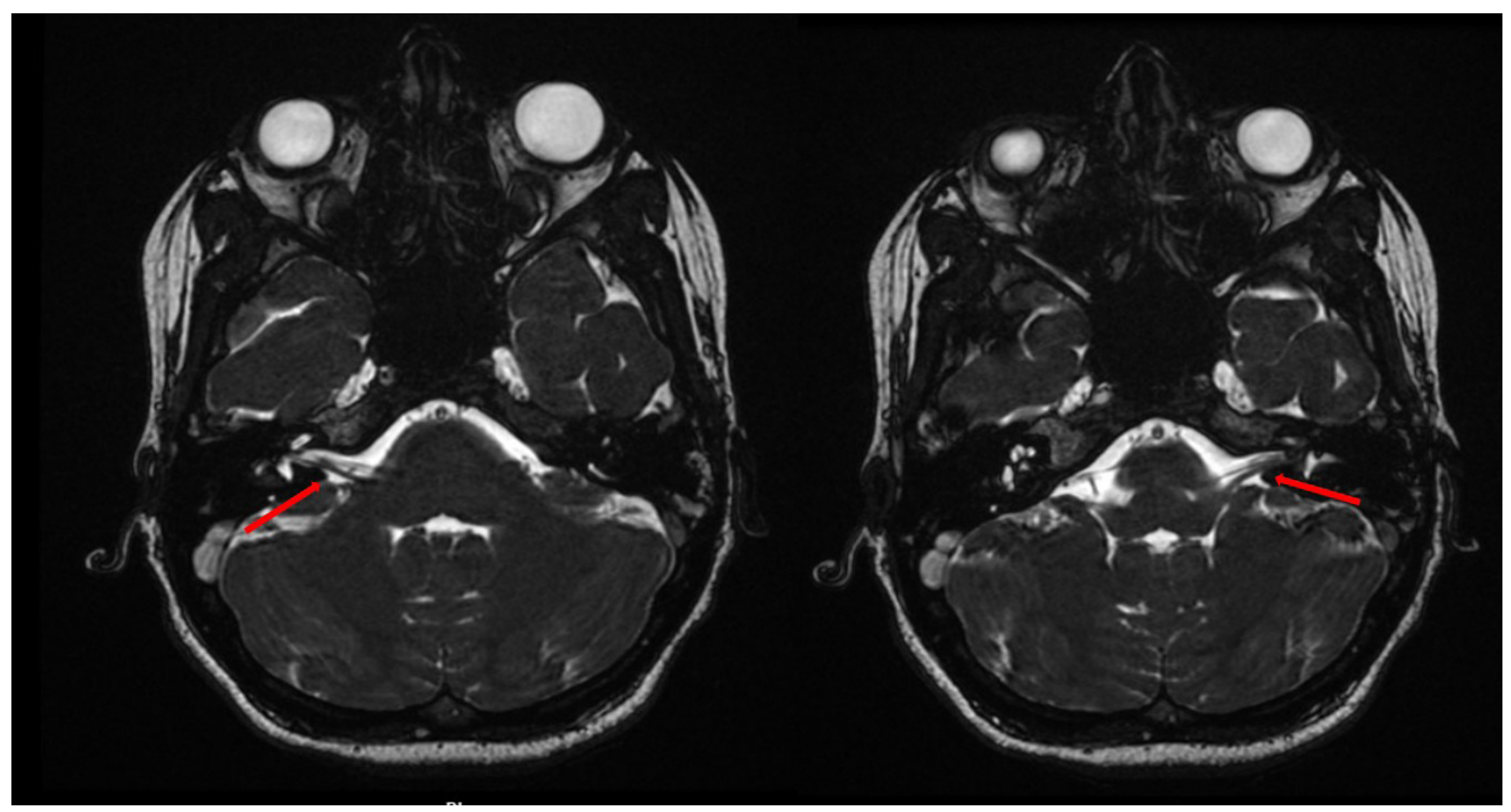

Figure 2. Magnetic Resonance Imaging of the brain showing normal trajectory of bilateral facial nerves. 
Table I. Various causes of facial palsy [4-8].

$\begin{array}{ll} & \text { Maxillofacial fractures } \\ \text { Trauma } & \text { Cranial fractures } \\ & \begin{array}{l}\text { Post-surgical palsy (base of skull, parotid } \\ \text { surgeries) }\end{array} \\ & \text { Herpes simplex virus 1 infection (HSV1) } \\ & \text { Epstein Barr virus infection } \\ & \text { Lyme's disease } \\ \text { Infections } & \text { Otitis media } \\ & \text { Meningitis } \\ & \text { Syphilis } \\ \text { Metabolic } & \text { Diabetes mellitus } \\ \text { disorders } & \text { Amyloidosis } \\ \text { Neurological } & \text { Stroke } \\ \text { disorders } & \text { Multiple sclerosis } \\ \text { Neoplastic } & \text { Parkinsonism } \\ \text { conditions } & \text { Intra cranial tumors } \\ & \text { Leukemia } \\ & \text { Guillian Barre syndrome } \\ \text { Syndromes } & \text { Ramsay Hunt syndrome } \\ & \text { Moebius syndrome } \\ & \text { Melkerson Rosenthal syndrome } \\ \text { Other causes } & \text { Heerfordt's syndrome } \\ & \text { Vasculitis }\end{array}$

Very few cases have been reported on transient bilateral facial palsy in young individuals. Siwula et al. reported a similar case of acute onset of facial palsy in a 6-year-old child [2]. Jairath et al. reported an Indian case series of Lyme disease affecting the skin in various presentations [9]. Systematic evaluation should be performed for finding out the central lesions causing bilateral facial nerve palsy.

In the present case, real-time PCR (Polymerase Chain Reaction) analysis was nonreactive for enterovirus, HSV 1\&2, paramyxovirus, and varicella zoster, which could result in transient bilateral facial weakness. MRI of the brain was normal, thereby ruling out central lesions causing facial palsy. Hence, after ruling out all the other possibilities for bilateral facial palsy, and correlating with the clinical, laboratory and radiological findings, a positive diagnosis of idiopathic bilateral Bell's palsy was hypothesized for the present case.
The uniqueness of the present case is that this is the first ever case of bilateral Bell's palsy in a young female which has been reported in the Indian population. Additionally, all the causes for bilateral palsy were thoroughly investigated, and this could probably represent a case of idiopathic bilateral Bell's palsy.

Cases of bilateral facial palsy often pose a diagnostic challenge. Though it could be due to several conditions, Lyme disease is one entity that diagnosticians need to consider in the list of differential diagnosis in cases of facial palsy in children.

\section{Acknowledgements}

The authors thank the patient and the patient's parents for their cooperation and Department of Neurology for their support.

\section{References}

1. Pothiawala S, LateefF. Bilateral facialnervepalsy: a diagnostic dilemma. Case Rep Emerg Med. 2012;2012:458371.

2. Jones B. Bell's palsy: an overview. InnovAiT. 2012:5(7):414419.

3. Siwula JM, Mathieu G. Acute onset of facial nerve palsy associated with Lyme disease in a 6 year-old child. Pediatr Dent. 2002;24:572-574.

4. Narayanan RP, James N, Ramachandran K, Jaramillo MJ. Guillain-Barré Syndrome presenting with bilateral facial nerve paralysis: a case report. Cases J. 2008;1:379. doi: 10.1186/1757-1626-1-379.

5. Jain V, Deshmukh A, Gollomp S. Bilateral facial paralysis: case presentation and discussion of differential diagnosis. $\mathrm{J}$ Gen Intern Med. 2006;21:C7-C10.

6. Gilden DH. Clinical practice. Bell's palsy. N Engl J Med. 2004;351:1323-1331.

7. Tiemstra JD, Khatkhate N. Bell's palsy: diagnosis and management. Am Fam Physician. 2007;76:997-1002.

8. Holland NJ, Weiner GM. Recent developments in Bell's palsy. BMJ. 2004;329:553-557.

9. Jairath V, Sehrawat M, Jindal N, Jain VK, Aggarwal P. Lyme disease in Haryana, India. Indian J Dermatol Venereol Leprol. 2014;80:320-323.

10. Biesiada G, Czepiel J, Leśniak MR, Garlicki A, Mach T. Lyme disease: review. Arch Med Sci. 2012;8:978-982. 